[Supporting Information to accompany J. Am. Chem. Soc. manuscript \# JA073034G-31-130]

\title{
Sharp Melting Transitions in DNA Hybrids Without Aggregate Dissolution: Proof of Neighboring-Duplex Cooperativity
}

\author{
Julianne M. Gibbs-Davis, George C. Schatz, and SonBinh T. Nguyen* \\ Department of Chemistry and International Institute for Nanotechnology, \\ Northwestern University, 2145 Sheridan Road, Evanston, Illinois, USA 60208-3113
}

\section{Experimental Section:}

General. UV-Vis spectra were recorded using a Hewlett-Packard (HP) 8452A diode-array spectrophotometer. Melting analyses of DNA-containing materials were performed using an HP 8453 diode-array spectrophotometer equipped with an HP 89090A Peltier temperature controller.

Materials. Polymer precursors were coupled to oligonucleotides following previously reported methodology. ${ }^{1}$ DNA strands were synthesized with an automated DNA synthesizer and CPGs (20-2031-10, dT-CPG 1000, $29 \mu \mathrm{mol} / \mathrm{g})$ from Glen Research (Sterling, VA). ${ }^{2}$ All starting materials for polymer synthesis and other reagents were purchased from Aldrich Chemicals, Lancaster Synthesis, or Alfa Aesar and used without further purification. Based on our published UVVis analysis of the polymer-DNA hybrids, ${ }^{3}$ the hybrids contain on average $\sim 5-6$ strands per polymer.

Thermal Denaturation Experiments. For the aggregate hybridization experiments, the concentration of each polymerDNA hybrid, expressed as [oligonucleotide], was $1.25 \mu \mathrm{M}$, and the concentration of $\mathrm{A}_{10}$ was $2.50 \mu \mathrm{M}$. The concentration of each type of oligonucleotide was $2.50 \mu \mathrm{M}$ for the polyDNA(T $\left.\mathrm{T}_{10} \mathrm{I}\right): \mathrm{A}_{10}$ experiment and $1.9 \mu \mathrm{M}$ for the polyDNA$\left(\mathrm{T}_{10^{-}}\right.$ I):polyDNA(T $\mathrm{T}_{10}$-II $)$ experiment. Solutions of oligonucleotides were prepared in PBS buffer $(1 \mathrm{~mL}, 0.3 \mathrm{M} \mathrm{NaCl}, 10 \mathrm{mmol}$ phosphate, $\mathrm{pH}=7.1$ ) and allowed to equilibrate overnight unless otherwise noted. For all the thermal denaturation curves, the hybridization mixtures were monitored in situ at $260 \mathrm{~nm}$ as a function of temperature $\left(1-{ }^{\circ} \mathrm{C}\right.$ intervals, 1 -min hold time, and a stir rate of $250 \mathrm{rpm}$ ). The resulting melting profiles were baseline-corrected by subtracting the average absorbance between 350 and $400 \mathrm{~nm}$.

For controls, we monitored the melting behaviors of unmodified $\mathrm{T}_{10}-\mathrm{I}: \mathrm{T}_{10}-\mathrm{II}: \mathrm{A}_{10}(1: 1: 2)$ mixtures, both annealed and unannealed, as well as the unmodified $\mathrm{T}_{10^{-}} 1: \mathrm{A}_{10}$ system. As expected for the former, the resulting melting profiles displayed two broad transitions with ascending temperature, corresponding to the melting of the $\mathrm{A}_{10}: \mathrm{T}_{10}$ duplex and the I:II duplex, respectively (Figure S1, red and blue lines), regardless of the thermal treatment. The two-strand $\mathrm{T}_{10}-\mathrm{I}: \mathrm{A}_{10}$ mixture only showed one transition at a lower temperature than both of the transitions for the $\mathrm{T}_{10}-\mathrm{I}: \mathrm{T}_{10}-\mathrm{II}: \mathrm{A}_{10}$ mixture, attributable to less base stacking in the $\mathrm{T}_{10}-\mathrm{I}: \mathrm{A}_{10}$ system (Figure S1, black line). ${ }^{4}$
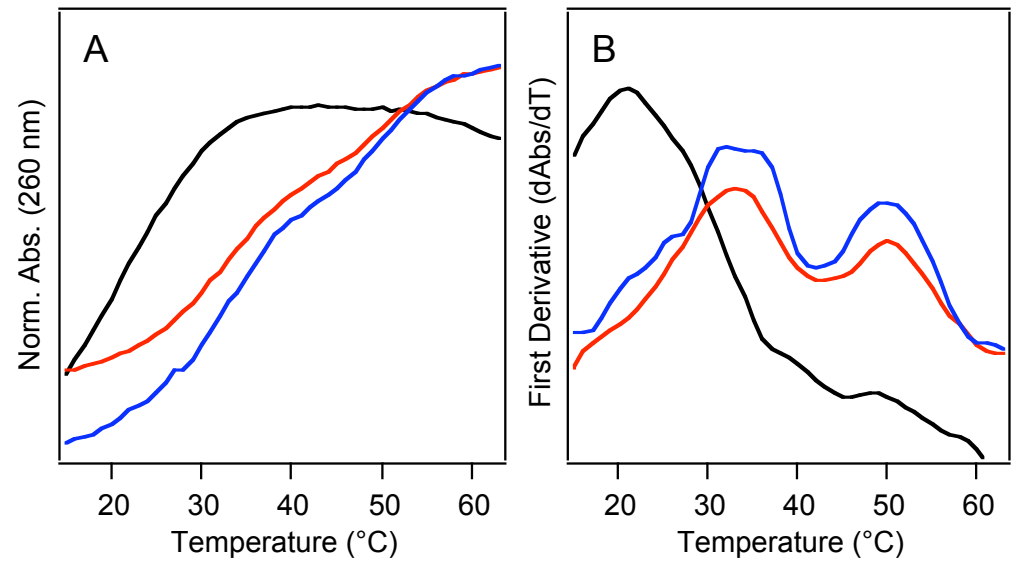

Figure S1. Melting profiles (A) and their corresponding first-derivative plots (B). Black line: $\mathrm{T}_{10}-\mathrm{I}: \mathrm{A}_{10}$ mixture $(5 \mu \mathrm{M}=$ total [DNA]). Red line: $\mathrm{T}_{10}-\mathrm{I}: \mathrm{T}_{10}-\mathrm{II}: \mathrm{A}_{10}$ mixture $\left(1: 1: 2 ; 5 \mu \mathrm{M}=\right.$ total [DNA]) annealed at $55^{\circ} \mathrm{C}$ for at least $8 \mathrm{~h}$ and then cooled to $10{ }^{\circ} \mathrm{C}$ overnight. Blue line: same mixture as red line but hybridized at room temperature for at least $8 \mathrm{~h}$ and then cooled to $10{ }^{\circ} \mathrm{C}$ overnight.

\footnotetext{
* Author to whom correspondence should be addressed. Email: stn@northwestern.edu and schatz@chem.northwestern.edu

${ }^{1}$ Watson, K. J.; Park, S.-J.; Nguyen, S. T.; Mirkin, C. A. J. Am. Chem. Soc. 2001, 123, 5592-5593.

${ }^{2}$ Brown, T.; Brown, D. J. S. In Oligonucleotides and Analogues; Eckstein, F., Ed.; Oxford University Press: New York, 1991.

${ }^{3}$ Gibbs, J. M.; Park, S.-J.; Anderson, D. R.; Watson, K. J.; Nguyen, S.; Mirkin, C. J. Am. Chem. Soc. 2005, 127, 1170-1178.

${ }^{4}$ Vasiliskov, V. A.; Prokopenko, D. V.; Mirzabekov, A. D. Nucleic Acids Res. 2001, 29, 2303-2313.
} 
Melting Profile Analysis. Igor Pro version 4.09A Carbon (Wavemetrics, Lake Oswego, Oregon) was used to process the measured melting data. The first derivatives of the melting curves were taken using the IntDiff XY Macros: differentiated, "splined", and extended via 300-point interpolation at the same $X$ values. A Gaussian curve of the form:

$$
Y_{o}+A \exp \left[-\left(\frac{x-x_{o}}{\text { width }}\right)^{2}\right]
$$

was then fit to the derivative (cursors were used to constrain the range for the profiles that exhibited two transitions) with the center, width, and normalization free to vary. The width was quoted in reference to the Gaussian width. The full-width at half maximum of the Gaussian is then FWHM $=2.3548\left(\text { width }^{2} / 2\right)^{0.5}$.

Prior to fitting, the temperature-axis was converted to Kelvin, and then the melting curves were corrected for changes in the UV-Vis signal that arise from the temperature-dependent absorptivity of single-stranded and double-stranded DNA. For experiments that exhibited only one melting transition, this is accomplished by fitting a straight line to the upper portion of the melting profile after the melting transition is complete and assigning that line to the absorbance of the singlestranded (i.e., fully dehybridized) DNA with temperature, $s(T)$ (Figure S2A). ${ }^{5}$ Similarly, the changes in duplex absorptivity not due to DNA melting are determined by fitting a line $d(T)$ to the portion of the melting profile before DNA melting starts. The dehybridized fraction $f$ can be determined from: ${ }^{6}$

$$
f=\frac{m(T)-d(T)}{s(T)-d(T)},
$$

where $m(T)$ is the UV-Vis absorbance melting profile.
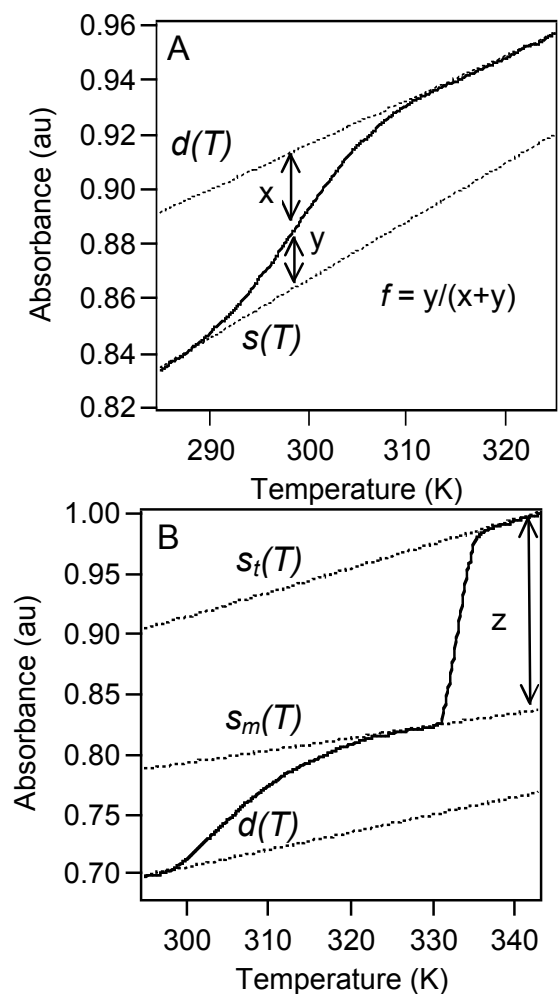

Figure S2. (A) Single-strand and duplex UV-Vis absorbance lines $(s(T)$ and $d(T)$, respectively) are extrapolated from the linear parts of the melting profile and used to calculate the fraction of duplex melting from the UV-Vis absorbance data. (B) For the three-component aggregate melting curves exhibiting two transitions, the singlestrand absorbance line $s(T)$ is determined from fits to the top and middle regions of the profile $\left(s_{m}(T)\right.$ and $s_{t}(T)$, respectively). The line fitting the middle portion of the profile $s_{m}(T)$ is first translated amount $\mathrm{z}$ in the $\mathrm{y}$ direction.

\footnotetext{
${ }^{5}$ Borer, P. N.; Dengler, B.; Tinoco, I., Jr.; Uhlenbeck, O. C. J. Mol. Biol. 1974, 86, 843-853.

${ }^{6}$ Marky, L. A.; Breslauer, K. J. Biopolymers 1987, 26, 1601-1620.
} 
To fit the melting profiles in experiments that displayed two melting transitions - corresponding to the $\mathrm{A}_{10}: \mathrm{T}_{10}$ and I:II dissociation events, respectively - the single-strand correction line $s(T)$ is a combination of linear fits to the middle and top portions of the melting profile. Specifically, for the first melting event, a line $s_{m}(T)$ is fit to the region in the middle of the melting profile (between the two melting events) and translated upward so as to intersect with the melting profile at the temperature that corresponds to the $\mathrm{UV}$-Vis absorbance maximum (Figure S2B). For the second melting event, a line $s_{t}(T)$ is fit to the linear region after the melting has finished. The single-strand correction line $s(T)$ is then assumed to be equal to $s_{m}(T)$ below the onset temperature of the second melting transition $\left(T_{l}\right)$ and equal to $s_{t}(T)$ at temperatures above the second melting transition $\left(T_{2}\right)$. Between $T_{1}$ and $T_{2}, s(T)$ is taken to be

$$
s(T)=\left(T_{2}-T\right)\left(\frac{s_{m}(T)}{T_{2}-T_{1}}\right)+\left(T-T_{1}\right)\left(\frac{s_{t}(T)}{T_{2}-T_{1}}\right) \quad \text { for } T_{1}<T<T_{2}
$$

The dehybridized fraction $f$ is then calculated using eq $\mathrm{S} 2$.

To determine the $\Delta H$ and $T_{m}$ for the control experiments that only exhibit one melting transition, an equation relating these thermodynamic parameters to the dehybridized fraction was derived in a similar fashion to that described by Jin et al. ${ }^{7}$ The equilibrium expressions for these two-component systems are

$$
\begin{array}{ll}
S=\left[\mathrm{Na}^{+}\right] & D_{M} \stackrel{K_{M}}{\rightleftharpoons} D_{M-1}+m S \quad \ldots \\
m=\text { number of } \mathrm{Na}^{+} \text {ions released } & D_{2} \stackrel{K_{2} \stackrel{K_{1}}{\rightleftharpoons} D_{1}+m S}{=} D_{0}+m S
\end{array}
$$

where $D_{0}$ and $D_{M}$ are the dehybridized and hybridized state, respectively. $M$ is the number of cooperative steps for the hybridization mixture (for the non-cooperative systems, such as polyDNA and unmodified DNA, $M=1$ ). The fraction of the fully dehybridized state $D_{0}$ can be expressed as:

$$
f=\frac{D_{0}}{D_{0}+D_{M}}=\frac{1}{1+\frac{1}{K}}
$$

The overall equilibrium constant $K$ is then a function of the binding constants corresponding to each step $\left(K_{i}\right)$ according to:

The van't Hoff equation for $K_{i}$ :

$$
\frac{1}{K}=\frac{S^{m M}}{K_{1} K_{2} \ldots K_{M}}
$$

$$
K_{i}=S^{m} \exp \left(\frac{-\Delta H_{i}}{R}\left(\frac{1}{T}-\frac{1}{T_{m, i}}\right)\right)
$$

can now be substituted into eq $\mathrm{S} 6$ and then applied in eq $\mathrm{S} 5$ to yield an expression relating $f$ to the thermodynamic parameters $\Delta H$ and $T_{m}$ :

$$
f=\frac{1}{1+\exp \left(\frac{\Delta H}{R}\left(\frac{1}{T}-\frac{1}{T_{m}}\right)\right)}
$$

\footnotetext{
${ }^{7}$ Jin, R.; Wu, G.; Li, Z.; Mirkin, C. A.; Schatz, G. C. J. Am. Chem. Soc. 2003, 125, 1643-1654.
} 